\title{
Je fais crise d'angoisse sur crise d'angoisse, de pire en pire : comment intensifier la peur dans le discours sur la « fin du monde »
}

\author{
After one anxiety attack I have another anxiety attack, \\ and it gets worst and worst: how to intensify/exaggerate \\ fear - discourse about the end of the world
}

\author{
Małgorzata Niziołek \\ Université Pédagogique de Cracovie \\ mniziolek1@gmail.com
}

\begin{abstract}
This paper analyses the comments of internet users related to the news about the end of the world on the 21 st of December, 2012. We consider which linguistic constructions allow fear to intensify. We indicate a semantic model of intensification which corresponds to the causative construction: Si Intens causes Si intens [very p (quality / condition)] causes [very q (act / new condition)], constituting the basis for the linguistic expression of fear.

Because the end of the world is an unimaginable event, it causes fear - a primary and universal emotion. Surfers exploit various (formal, lexical, graphic, etc.) procedures which provide an opportunity for the feelings of fear to intensify. The intensification of the cause (approaching end of the world) provokes an intensification of the consequence (feeling of fear). The analysis is part of the work of the research group DiSEM (Discourse Semantics Inference) composed of researchers from the Pedagogical University of Cracow.
\end{abstract}

Keywords: intensification, linguistic constructions of exorbitance/intensification, analysis of discourse, linguistic concept of fear, the end of the world

\section{INTRODUCTION}

Le 21 décembre 2012 est l'une des dates dont de nombreuses personnes se souviendront encore longtemps. La fin du monde, prévue ce jour-là, avait été très médiatisée. La sortie du film 2012 a encore amplifié la discussion autour de ce sujet très prenant. Internet est devenu le lieu privilégié de la naissance de différents fantasmes sur cette nouvelle prophétie. Et comme l'humanité cherche 
régulièrement à se faire peur, la peur est devenue un des sujets essentiels dans les discussions sur le Web. L'important est moins que la fin du monde survienne vraiment que le fait que certains y croient, qu'on en parle. Le réseau a permis aux gens d'exprimer leur peur liée à cet événement attendu et appréhendé.

$\grave{A}$ mesure que la date fatidique approchait, le nombre des commentaires de plusieurs internautes augmentait. Nombreux étaient ceux qui voulaient discuter/partager leurs émotions/craintes de la fin du monde avec les personnes à qui elle faisait peur. Comment réagissent les peureux, ceux et celles qui ne peuvent pas s'empêcher d'y croire tout au fond d'eux ? Comment leur peur s'exprime à travers les commentaires ? ${ }^{1}$

Dans cet article, nous nous proposons d'étudier des procédés langagiers qui ont été utilisés pour intensifier/exagérer la peur. Il s'agit avant tout de récupérer des assertions trop informatives ou celles qui renferment une quantité excessive d'information. On est là au cœur de la notion d'excès, d'exagération. On définit l'excès comme "fait, acte d'aller au-delà de ce qui est permis, convenable au regard de toutes sortes de normes, de la morale, de l'esthétique ou des convenances sociales » (TLF). En général, l'excès renvoie à un dépassement de mesure. Nous allons nous concentrer sur les moyens linguistiques qui provoquent ce dépassement de mesure: «Lorsque nous sommes vivement frappés de quelque idée que nous voulons représenter, et que les termes ordinaires nous paraissent trop faibles pour exprimer ce que nous voulons dire, nous nous servons de mots qui, à les prendre à la lettre, vont au-delà de la vérité, et représentent le plus ou le moins, pour faire entendre quelques excès en grand ou en petit » (Dumarsais, $1988: 131$ ).

Dans notre analyse, nous adoptons donc la définition élargie de l'exagération, de l'excès.

L'intensification renvoie à des phénomènes hétérogènes. Elle concerne le niveau lexical (p.ex. préfixes de haut degré, gradation lexicalisée d'une propriété, etc.), le niveau sémantico-syntaxique (exclamative et haut degré, comparaison à un parangon, etc.), le niveau prosodique (intonation, etc.), le niveau énonciatif et argumentatif (Anscombre \& Tamba, 2013 : 3). De nombreuses études concernant ce domaine de recherche se multiplient depuis une dizaine d'années. Romero $(2001,2004,2007)$ décrit différents moyens de s'exprimer intensément en français. Pilecka (2010) propose une analyse approfondie des verbes intensifieurs en français. Dans cet article, nous voulons nous concentrer surtout sur la structure causale qui est à la source de l'intensification et analyser des procédés utilisés pour intensifier la peur.

${ }^{1}$ L'article s'inscrit dans les travaux du groupe de recherche DiSem (Discours Inférence Sémantique) composé de chercheurs de l’Université Pédagogique de Cracovie (Muryn, Niziołek, Hajok, Prażuch). 


\section{CORPUS DE RECHERCHE ET SES CARACTÉRISTIQUES}

Puisque tout genre de texte entraîne des contraintes, des limites et des prescriptions, il fallait délimiter un corpus assez homogène qui garantisse la présence des formules recherchées. Dans notre étude, le corpus que nous avons exploité compte 126000 mots et il contient des commentaires des internautes inspirés par des articles sur la fin du monde extraits des sites suivants ${ }^{2}$ :

- http://www.notre-planete.info/actualites/actu_2175_2012_fin_du_monde.php

- www.sos-2012.com

- http://www.211212.info

- http://forum.doctissimo.fr/psychologie/angoisse-phobie/monde-2012-sujet _158800_1.htm

- http://www.espritdavant.com/DetailElement.aspx?numStructure $=79255 \&$ numElement $=102250 \&$ numRubrique $=462379$

- http://www.slate.fr/life/65429/la-fin-du-monde-et-la-verite-sur-le-calendriermaya?page $=3$

\subsection{FIN DU MONDE DANS LE CORPUS}

Le concept de fin du monde apparaît sous plusieurs expressions qui reflètent l'attitude des internautes à l'égard du phénomène. Mais comment prendre au sérieux quelque chose qui n'est qu'une légende, une supercherie ou une ruse. On peut diviser les noms utilisés pour parler de la fin du monde en 4 groupes :

- une légende, un mythe, la prophétie, une soi-disant prédiction;

- des bruits, des rumeurs, des rumeurs, rien que des rumeurs, des bobards, des balivernes ;

- un/des mensonge(s), un ENOOORME MENSONGE, une ruse, une supercherie, une ânerie, une vaste bêtise, des idioties, une stupidité, un délire, une absurdité, du blabla, une fumisterie, une blague, une big jok, une mauvaise blague, des bêtises, une vaste connerie, du grand n'importe quoi, histoire à dormir debout, un fantasme, l'hérésie pure, une histoire de dingue ;

- l'apocalypse, une date fatidique.

Le premier groupe, le moins représenté, montre la fin du monde comme un récit imaginaire, dont la fonction principale est de représenter symboliquement

\footnotetext{
${ }^{2}$ Le choix d'un autre corpus, p.ex. de la nouvelle fantastique, nous donnerait des résultats tout à fait différents.

${ }^{3}$ Nous gardons la forme originale dans tous les exemples parce qu'elle véhicule des informations supplémentaires.
} 
le cycle de vie et différents événements. C'est quelque chose qui fonctionne dans l'imaginaire collectif et n'est qu'une représentation métaphorique. Les substantifs du deuxième groupe mettent en question la véracité de cet événement. Les substantifs du troisième groupe mettent l'accent sur le côté mensonger de l'information sur la fin qui s'approchait. Il s'agit d'une histoire inventée et non confirmée, diffusée pour tromper un public généralement crédule.

Seules « l'apocalypse » et « une date fatidique » renvoient directement à la catastrophe qui s'approche. Aucune de ces co-occurrences ne renvoie à une information confirmée, sérieuse qui pourrait provoquer une peur/angoisse. L'idée de mensonge, de blague est un des traits dominants dans la plupart de ces substantifs. Pour cette raison, le sentiment de peur, exprimé à plusieurs reprises dans les commentaires, n'est pas fondé.

\subsection{PEUR : ENTRE CROIRE ET NE PAS CROIRE}

De nombreuses personnes éprouvent la peur face à l'annonce de la fin du monde. Ce qui est intéressant, ce sont les réactions des gens à cette annonce de la fin des temps : même s'ils savent que ce ne sont que « des balivernes", ils commencent à y croire ou même s'ils n'y croient pas, commencent à en avoir peur.

En général, on observe trois attitudes/réactions (apparemment opposées) à l'annonce de la fin du monde :

\section{a) Ceux qui croient et ont peur}

(1) Oui j'y crois, oui j'ai peur

(2) Personnelement, J'y crois et j'y crois pas et je regarde tout le temps le compte à rebourd et j'ai Bcp Peur !! =((

b) Ceux qui ne croient pas et ont peur

(3) moi je croie pas mais j'ai peur

(4) Personelement j'y croit pas mais j'ai peur

(5) Moi perso j'y croit pas sa me fait quand même un peu peur

(6) J AVAIS QUAND MEME PEUR MAIS JE SAIS QUE CE N EST PAS VRAIS

c) Ceux qui ne croient pas

(7) pour moi chers amis je ne crois a cette fameuse fins du monde cet clair et net

(8) Moi j y crois pas

(9) C Faux! C faux! C faux! 


\section{STRUCTURE CAUSALE À LA SOURCE DE L'INTENSIFICATION/EXAGÉRATION}

Dans cette situation, il est intéressant de voir la structure causale qui est à la source des réactions des internautes (dans la plupart des cas exagérées) face à la fin du monde.

Parmi les structures où l'intensité/l'exagération peut être considérée comme élément dominant, c'est certainement celle où le dépassement de mesure est perçu comme changement par rapport à une norme (subjective, objective, peu importe) et entraîne une réaction. Il s'agit donc d'une structure causale que nous pouvons paraphraser après S. Karolak (2001 : 152) comme il est arrivé $p$ qui $a$ entraîné $q$ (où $p$ et $q$ sont des propositions) et qui, dans notre cas, aurait la forme de $p$ est tel qu'il a entraîné q, où tel (si intense ou si fréquent) semble focalisé dans la composante antécédente. Une telle structure peut avoir plusieurs réalisations syntaxico-rhétoriques, mais celle qui est la plus adéquate c'est le système consécutif. La construction consécutive analysée a, dans le discours, la forme suivante: +Intens $(p)$ cause +Intens $(q)$ et le modèle [très/trop $p$ (qualitélétat)] cause [très/trop q (acte/nouvel état)] est une des réalisations de la structure prototypique (Muryn, Niziołek, Prażuch, Hajok, sous presse). Dans notre perspective, l'intensification est un prédicat d'ordre supérieur aux arguments propositionnels ( $p$ et $q$ ).

Dans le corpus analysé, cette structure peut être représentée de façon suivante :

$p$

$q$

[la fin du monde approche] est pour moi inimaginable CAUSE [j'ai peur] terriblement

[SI]

[SI]

L'intensification est un jugement subjectif porté sur une occurrence particulière d'une proposition, ici [la fin du monde approche]. Ce jugement n'est qu'une opinion momentanée de celui qui la prononce dans un contexte bien précis et qui peut ne pas être partagée par son interlocuteur. Dans la structure cidessus, ce jugement subjectif se superpose sur [la fin du monde approche]. Cet événement est inimaginable pour moi (par exemple Moi perso j'y croit pas sa me fait quand même un peu peur). Les causes de la peur sont personnalisées. Les internautes focalisent sur leur propre perception de la fin du monde (moi, je, perso, etc.).

Face à un événement extrême $-p$, ici la fin du monde, la réaction $-q$, la peur, peut/doit être extrême. Plus la cause de la peur est grande plus la ré- 
action à cette cause s'intensifie. L'intensité de la cause infère l'intensité de la conséquence, plus la cause est intense, plus sa conséquence est intense. La fin du monde fait peur parce qu'elle est synonyme de la mort, de l'anéantissement, du pire (même si dans les commentaires, on met l'accent sur le côté mensonger de cet événement).

Revenons à la cause. La peur est irrationnelle parce qu'elle manque de vraie cause. On porte un jugement subjectif sur l'annonce de la fin des temps par les Mayas. On a peur parce qu'on se dit que les Mayas étaient peut-être les seuls à connaître les vérités cachées, ils savaient plus que nous et cela nous fait encore plus peur. Mais est-ce que cette source est fiable? Face à des arguments (à vrai dire nuls) qui visent à confirmer/expliquer la fin du monde, on ne peut pas réagir de façon raisonnable en passant par une simple argumentation/déduction. Les réactions à cette cause non-confirmée sont donc exagérées. Des arguments qui apparaissent pendant les discussions, souvent acharnées, ne sont pas fondés.

\section{EXPOSANTS DE L'INTENSIFICATION}

Nous ne voulons pas nous attarder sur la notion de peur qui a déjà été analysée en détail dans plusieurs études. Il faut juste rappeler que la peur est une émotion primaire. Dans la langue française, plusieurs noms sont utilisés comme synonymes ou sémantiquement apparentés pour exprimer le concept de peur. Dans notre corpus, les synonymes de la peur sont presque inexistants (il faut souligner qu'il n'existe pas de corrélation parfaite entre deux mots et la synonymie absolue n'existe pas). On repère aussi des mots qui appartiennent au registre familier par exemple la trouille, la frousse ou le verbe flipper.

La peur peut être analysée selon différents critères dichotomiques. Bresson et Dobrovolski en énumèrent huit (1995: 108) :

(1) fort vs faible

(2) attente de quelque chose de mauvais vs réaction à quelque chose de mauvais

(3) "personnel » (le sujet psychologique lui-même ou son entourage est concerné) vs « général»

(4) contrôle vs perte du contrôle de son comportement

(5) soudaineté vs caractère moins soudain

(6) duratif vs bref

(7) courant vs rare ou plus rare

(8) stylistiquement neutre vs stylistiquement marqué

Cependant, nous allons nous concentrer seulement sur deux critères, à savoir l'intensité de la peur (1) et son expression dans la langue (8).

La constatation qui est le point de départ de l'analyse est que la peur est déjà un sentiment intense. Au sein du domaine de la peur, on distingue des unités 
à une plus forte intensité. Ainsi, la frayeur ou la panique sont-elles plus intenses que la peur tandis que la crainte l'est moins. Les exposants stylistiques de la peur subissent eux-mêmes une gradation. Cependant, l'analyse contextuelle nous fournit des exemples où la peur devient très intensive. Quels sont donc les procédés linguistiques/langagiers qui permettent d'intensifier le sentiment de la peur ? ${ }^{4}$

Sur le plan de l'expression, l'intensification peut avoir des exposants explicites ou fonctionner de façon plus ou moins implicite.

\subsection{EXPOSANTS FORMELS DE DEGRÉ D'INTENSITÉ}

Parmi les exposants formels on peut classifier entre autres très, beaucoup, trop. Très est considéré comme purement intensif et beaucoup peut désigner tant la quantité que l'intensité. Ces trois indicateurs d'intensité forte servent à décrire les expressions de haut degré. On les retrouve souvent dans notre corpus. En voici quelques exemples :

(10) j'ai très peur de la fin du monde comment faire pour pensée a autres chose ????

(11) o les gas j'ai trop peur!!!!

(12) j'ai Bcp Peur!!

Le même degré élevé d'intensité est véhiculé par des adverbes en -ment, par exemple horriblement.

(13) J'ai horriblement peur.

La répétition est un autre moyen utilisé par des internautes pour marquer l'exagération, pour intensifier le sentiment qu'ils ressentent. La répétition peut concerner soit un terme simple, soit un groupe syntaxique.

Dans les exemples (14) et (15), par le redoublement de l'intensifieur très et trop, les interlocuteurs marquent une intensité plus forte que si on l'avait énoncée : J'ai très peur ou j'ai trop peur.

(14) J'ai très très peur

(15) sérieux j'ai trop trop peur

Il arrive que les locuteurs répètent un syntagme dans son intégralité. Cette répétition a pour effet de faire ressortir le discours par rapport au reste du texte. Dans (16) et (17) la peur apparaît comme un sentiment dominant, voire obsessionnel.

\footnotetext{
${ }^{4}$ Certains exemples peuvent apparaître deux ou même trois fois parce que l'exagération s'opère sur plusieurs éléments de la phrase.
} 
(16) jai peur de la fin du monde en 2012 car je nest que 10 ans je veut vivre moi je ne veut pas perdre ma famille je ne veut pas perdre mes copines jai peur jespere que se nest pas en 2012 la fin du monde tous les soirs je pleure tellement jai peur je nest pas envie de mourir meme si un jour on va mourir jai peur

(17) je n arrive pas a pas y croire!! jai tellement peur tout le monde disens que la fin du monde c dans deux jours!!!le vzndredi 21 decembre 2012!!j ai tellement peur

Il est important de différencier les répétitions d'unités de signification des étirements graphiques, en particulier lorsque ces premières sont agglutinées. On parle d'étirements graphiques quand la séquence reproduite est un signe graphique, alors qu'il y a répétition lorsque la séquence reproduite est supérieure à un signe graphique ou à une unité minimale de signification. Ainsi, la séquence $b c p$ reproduite dans l'exemple suivant est unrépétition :

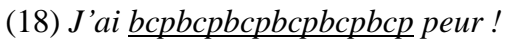

\subsection{SENS MÊME DES MOTS AU SERVICE DE L'INTENSIFICATION}

Il existe des verbes/adjectifs/substantifs qui sont sémantiquement plus marqués par rapport à la peur. Le degré élevé d'intensité de la peur est contenu dans le sens même de ces notions.

La panique est un des rares synonymes de la peur qui soit utilisé par les internautes. On définit la panique comme " peur, terreur qui survient de manière subite et violente en troublant l'esprit et le comportement. Vive terreur, soudaine et irraisonnée, souvent dénuée de fondement qui affecte le plus souvent un groupe ou une foule et provoque de grands désordres » (TLF).

L'énoncé (19) est plus marqué sur le plan de l'intensification. Le verbe paniquer, un verbe au sémantisme fort, est encore augmenté dans (20) par l'intensifieur plus.

(19) Je panique, c'est le mot exacte.

(20) Il disais qu il allait se passer a la cote belge un enorme tsunami avant le 21 decembre alors $j$ ai encore plus panique

(21) La fin approche, le gouvernement ne dit rien sur le sujet de peur et la population panique.

Pas étonnant que autant de gens paniquent lorsque hollywood met en scène la fin des temps (mourir quoi!)

La terreur est «le synonyme » suivant de la peur. La terreur est une peur extrême, angoisse profonde, une très forte appréhension saisissant quelqu'un en présence d'un danger réel ou imaginaire. Le substantif la phobie (22) dévoile 
encore un trait de la peur. Le sentiment de peur qu'on ressent est irraisonné ou instinctif.

(22) moi j'ai pas cette phobie, je comprend les gens qui vivent dans cette terreur.

Le cauchemar qui apparaît dans (23) désigne un rêve pénible ou effrayant qui réveille le dormeur en le laissant dans un malaise ou dans l'angoisse (TLF).

(23) Moi cela m' a inquiété et j' en ai fait des cauchemars avec ça

\subsection{MANIFESTATIONS DE LA PEUR}

On sait très bien que la peur s'exprime à travers différentes manifestations (universelles et stéréotypées) corporelles et comportementales. On énumère souvent : les mains moites, la dilatation des pupilles, etc. Dans notre corpus la peur est représentée par les manifestations suivantes :

- Se cacher :

(24) c'est fou ce que ca provoque chez les gens une rumeur de la fin du monde, des gens $\underline{\text { creusent et construisent des bunkers }}$ d'autre cherchent à se repentir

- Pleurer :

(25) j'ai peur jespere que se nest pas en 2012 la fin du monde tout les soirs je pleure tellement que jai peur

Ce ne sont pas des peurs occasionnelles mais obsessionnelles.

- Frissons :

(26) Est ce que toute cette histoire est vraie? Répondez S.V.P. Je grelote de peur.

- Insomnies :

(27) Vous allez me prendre pour une débile mais j'en dors plus de la nuit ..ça me rend malade cette histoire :-(

(28) sérieux j'ai trop trop peur j'ai carrément des insomnies

- Mal au ventre :

(29) Moi j ai vraiment peur que j’en ai mal au ventre tous les jours et que jai du mal à en dormir

Quand les manifestations se prolongent dans le temps, elles deviennent permanentes et peuvent se transformer en troubles psychiques. 
- Les troubles anxieux et la dépression :

(30) j'angoisse, je fais des crises de panique, je suis sous ANTI Dépresseurs, Je regarde mes 3 garçons avec la peur au ventre, ils ont 16-13 ans et 4mois... je pleure sans cesse, j'ai horriblement peur, j'ai peur de faire des conneries la veille...

(31) moi j'ai très peur je n'en dors plus, je mange plus, je pleures tous le temps! c'est horrible!

(32) Je fais crise d'angoisse sur crise d'angoisse, de pire en pire

Le (32) est un des exemples où l'intensification de la peur atteint son paroxysme.

\subsection{PROCÉDÉS GRAPHIQUES DE L’EXAGÉRATION}

L'intensification peut s'inscrire également dans la façon d'écrire. Elle se manifeste au niveau graphique sous plusieurs formes. Parmi les procédés graphiques qui sont mis en œuvre pour mettre un élément en relief on observe, entre autres, emploi de majuscules, étirements graphiques ou smileys. La simplicité du procédé typographique manifeste cette accentuation.

\section{- Emploi de majuscules}

Les (33), (34), (35) sont des phrases banales, très simples qui n'apportent rien de nouveau à ce qui a déjà été dit. Cependant l'emploi de majuscules pour le verbe mourir dans (34) ou la collocation avoir peur attestent l'importance du contenu véhiculé par ces énoncés aux yeux des émetteurs des messages.

(33) ÇA FAIT PEUR

(34) ON VA TOUS MOURIR on va pas mourir (gros mot) jai peur !!!!!!!!!!!!!!!!!!!!!!!!!!!!!!!!!!!!!!!!!!!!!!!!!!!!!!!!!!!!!!!!!!!!!!!!!!!!!!!!!!!!!!!!!!!! !!!!!!!!!!!!!!!!!!!!!!!!!!!!!!!!!!!!!!!!!!!!!!!!!!!!!!!!!!!!!!!!!!!!!!!!!!!!!!!!!!!!!!!!!!!!!! !!!!!!!!!!!!!!!!!!!!!!!!!!!!!!!!!!!!!!!!!!!!!!!!!!!!!!!!!!!!!!!!!!!!!!!!!!!!!!!!!!!!!!!!!!!

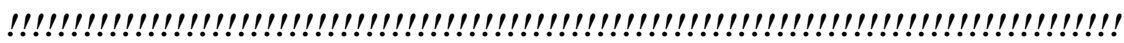

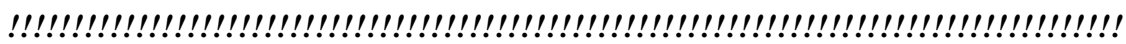
!!!!!!!!!!!!!!!!!!!!!!!!!!!!!!!!!!!!!!!!!!!!!!!!!!!!!!!!!!!!!!!!!!!!!!!!!!!!!!!!!!!!!!!!!! !!!!!!!!!!!!!!!!!!!!!!!!!!!!!!!!!!!!!!!!!!!!!!!!!!!!!!!!!!!!!!!!!!!!!!!!!!!!!!!!!!!!!!!!!!!!!! !!!!!!!!!!!!!!!!!!!!!!!!!!!!!!!!!!!!!!!!!!!!!!!!!!!!!!!!!!!!!!!!!!!!!!!!!!!!!!!!!!!!!!!!!!!!! !!!!!!!!!!!!!!!!!!!!!!!!!!!!!!!!!!!!!!!!!!!!!!!!!!!!!!!!!!!!!!!!!!!!!!!!!!!!!!!!!!!!!!!!!!!!!!!!! !!!!!!!!!!!!!!!!!!!!!!!!!!!! : ( : J ai trop peur arretez (gros mot) !!!!!!!!!!!!!

(35) j'ai Bcp Peur !! =((

Dans (35), la lettre B en majuscule fait ressortir l'intensifieur par rapport au reste du texte. C'est encore un moyen qui permet de transcrire l'intensité. 


\section{- Etirements graphiques}

Les internautes ne recourent pas souvent aux étirements graphiques. Nous n'en avons repéré que quelques exemples. Des étirements graphiques (l'allongement) sont utilisés pour insister sur un élément du discours ou pour transcrire la prononciation d'une séquence linguistique. Nous distinguons deux types d'étirements graphiques : le premier consiste à répéter une ou plusieurs lettres dans une séquence graphique (36) et (37), et le second consiste à répéter un signe de ponctuation (36). Voici des exemples illustrant les deux phénomènes :

(36) J'AI PEUR aaaaaaaaaaaahhhhhhhhhhhhhhhhhhhh !!!!!!!!!!!!!!!!!!!!!!!!!!!!!!!!!!!!!!!!!

(37) J'ai trop peur je veut devenir chanteuse en + et je ne pourrais pas réaliser mon rêve j'ai vraiment trop peur la il reste que 16 jours !!! :( on vas touиuииusssss mourriirrrrrrrrrr)

\section{- Smileys (binettes)}

Les smileys ou binettes sont une expression graphique des émotions. Leur rôle est de renforcer la valeur expressive présentée dans le contenu verbal. Dans les exemples cités, il y a une redondance entre la dimension expressive des smileys et le contenu verbal. La dernière binette dans (39) tranche sur l'interprétation à donner. On pourrait supposer, sans binette, que la personne qui l'écrit n'a pas peur (pour rien). Or, la binette ne laisse pas d'illusions, les émotions l'emportent sur la raison.

(38) oui j'y croit, oui j'ai peur Oh mon dieu pourquoi si tot? :'(

(39) J'ai peur 9 très peur....je ne dois pas y croire mais je ne sais pas...cette semaine là va passer vite...et ce sera Vendredi... je stresse mais je devrais pas.. (1) Je ne sais pas si c'est vrai ou faux toute cette fin du monde...j'espere juste que ces fichus mayas ont tords car je ne veux pas mourir encore moins j'ai peur d'etre la seule survivante...je sais c'est bete mais je me fais des idées noires pour rien 9

\subsection{PHRASE EXCLAMATIVE}

La phrase exclamative est un moyen d'expression de l'intensité par excellence. La prolifération des points d'exclamation met en valeur l'intensité de la peur ressentie par les internautes. Le point d'exclamation représente le discours sous une forme écrite, il est chargé d'une valeur affective que n'ont pas les autres signes de ponctuation. Comme son emploi est souvent facultatif, il dépend avant tout de la volonté de l'auteur. Et les auteurs des commentaires font souvent recours à ce moyen pour mettre en relief leur sentiment, parce qu'ils ne 
trouvent pas d'autres moyens pour dire à quel point l'annonce de la fin du monde leur fait peur.

(40) ON VA TOUS MOURIR on va pas mourir (gros mot) jai peur !!!!!!!!!!!!!!!!!!!!!!!!!!! !!!!!!!!!!!!!!!!!!!!!!!!!!!!!!!!!!!!!!!!!!!!!!!!!!!!!!!!!!!!!!!!!!!!!!!!!!!!!!!!!!!!!!!!!!!!!!!!!!!!!!!!!!!!!!!!! !!!!!!!!!!!!!!!!!!!!!!!!!!!!!!!!!!!!!!!!!!!!!!!!!!!!!!!!!!!!!!!!!!!!!!!!!!!!!!!!!!!!!!!!!!!!!!!!!!!!!!!!!!!! !!!!!!!!!!!!!!!!!!!!!!!!!!!!!!!!!!!!!!!!!!!!!!!!!!!!!!!!!!!!!!!!!!!!!!!!!!!!!!!!!!!!!!!!!!!!!!!!!!!!!!!!!!!!!! !!!!!!!!!!!!!!!!!!!!!!!!!!!!!!!!!!!!!!!!!!!!!!!!!!!!!!!!!!!!!!!!!!!!!!!!!!!!!!!!!!!!!!!!!!!!!!!!!!!!!!!!!!!! !!!!!!!!!!!!!!!!!!!!!!!!!!!!!!!!!!!!!!!!!!!!!!!!!!!!!!!!!!!!!!!!!!!!!!!!!!!!!!!!!!!!!!!!!!!!!!!!!!!!!!!!!!!!! !!!!!!!!!!!!!!!!!!!!!!!!!!!!!!!!!!!!!!!!!!!!!!!!!!!!!!!!!!!!!!!!!!!!!!!!!!!!!!!!!!!!!!!!!!!!!!!!!!!!!!!!!!!!!! !!!!!!!!!!!!!!!!!!!!!!!!!!!!!!!!!!!!!!!!!!!!!!!!!!!!!!!!!!!!!!!!!!!!!!!!!!!!!!!!!!!!!!!!!!!!!!!!!!!!!!!!!! !!!!!!!!!!!!!!!!!!!!!!!!!!!!!!!!!!!!!!!!!!!!!!!!!!!!!!!!!!!!!!!!!!!!!!!!!!!!!!!! :( : J ai trop peur arretez (gros mot) !!!!!!!!!!!!!!

(41) c'est horrible!

L'utilisation d'onomatopées et d'interjection introduit également des humeurs de façon symbolique, renforçant l'expressivité en discours.

(42) НААААААААААААААААААНАНАНАННАНАНАННАНАНАННАНАННАНАНН АНАННАНАННА !!!!!!

(43) aaaaaaaaaaaaaaaaaaaaaaaaaaa .... sa me stresse

\subsection{INTENSIFICATION INFÉRÉE}

L'intensification inférée est un phénomène absent des ouvrages consacrés à l'intensification. Cependant, nous avons pu voir que les moyens formels ne sont pas les seuls à décider du degré d'intensité du sentiment de la peur.

Dans (44), la peur est à inférer du deuxième élément de la phrase qui est en contradiction avec le début du commentaire. Même si l'internaute avoue ne pas avoir peur, il cherche le soutien de sa famille pour se rassurer, pour se réconforter.

(44) J'ai pas peur de la fin du monde je veux voir ma famille !!! Bouh !!!

\section{CONCLUSION}

L'analyse de notre corpus montre qu'il n'est pas important ce que les gens disent, mais ce qu'ils ressentent. A la source des réactions émotives des gens, il y a une structure conceptuelle qui se base sur des vérités communément acceptées et le savoir partagé est un de ses traits définitionnels. Comme la fin du monde est un événement inimaginable, elle provoque la peur qui est un sentiment primaire et universel. L'intensification de la cause (l'annonce de la fin 
du monde) provoque l'intensification de la conséquence (peur). La peur se manifeste dans les commentaires des internautes à travers différents exposants mais les procédés graphiques 1'emportent. Les internautes éprouvent un grand besoin de partager l'angoisse qui les tourmente mais ils ne savent pas comment le faire, il leur manque des moyens pour l'exprimer. Tous les exposants de l'intensification décrits sont des réalisations du modèle sémantique de l'intensification. 


\section{BIBLIOGRAPHIE}

Anscombre, J.-C., TAMBA, I. (2013): «L'intensification ». Langue française, 177.

BogACKI, K., KAROLAK, S. (1991): «Fondements d'une grammaire à base sémantique ». Lingua e Stile, XXVI, 309-345.

Bourion, E. «Le réseau associatif de la peur ». CNRS, InaLF, Nancy (http://www.revue-texto. net/Parutions/Analyse-thematique/Bourion.pdf).

Bresson, D., Dobrovol'skiJ D. (1995): «Petite syntaxe de la peur. Application au français et à l'allemand ». Langue française, 105, 107-119.

DuMARSAIS (1988): Des tropes ou des différents sens. Paris : Flammarion.

FERRE, G. (2004): « Degrés d'intensité exprimés à l'oral. Du discours à la gestualité en passant par la prosodie ». In : M. Noailly \& F. Lefeuvre, 13-26 (hal-00135441).

KAROLAK, S. (2001): Od semantyki do gramatyki. Warszawa: SOW.

KAROLAK, S. (2007): Sktadnia francuska o podstawach semantycznych, t. 1. Kraków: Collegium Columbinum.

IZERT, M. (2002): Les expressions Adj comme SN et l'intensification de la propriété, thèse de doctorat.

Muryn, T. (2005): « Continuité, intensité et fréquence ». Synergies Pologne, 1, 78-84.

Muryn T., Niziolek M., Hajok A., Prazuch W. (sous presse), Ce café me réveille ou l'intensité inférée.

Nevzorova, S. (2011): "Sur l'expression verbale de l'émotion dans le contexte culturel». Romanica Cracoviensia, 11, 300-307.

PILECKA E. (2010): Verbes intensifieurs et leur fonctionnement en français contemporain. Lask: Oficyna Wydawnicza Leksem.

Riegel M., Pellat J.-Ch., Rioul R. (2001): Grammaire méthodique du français. Paris : Presses Universitaires de France.

ROMERO, C. (2001): L'intensité en français contemporain : analyse sémantique et pragmatique, thèse de doctorat, Université Paris 8 (http://marg.lng2.free.fr/documents/the 0013 romero c/the0013.pdf).

Romero, C. (2004): « Nouvelles remarques sur l'hyperbole». In : M. H. Araujo Carreira (ed.), Plus ou moins ?! L'atténuation et l'intensification dans les langues romanes/ Travaux et Documents, 24. Saint Denis : Presses Universitaires de Vincennes, 265-282.

Romero, C. (2007): «Pour une définition générale de l'intensité dans le langage ». Travaux de linguistique, 54, 40-46.

STRAŚ, E. (2008): Kategoria intensywności we frazeologii. Katowice: Wydawnictwo Uniwersytetu Śląskiego. 